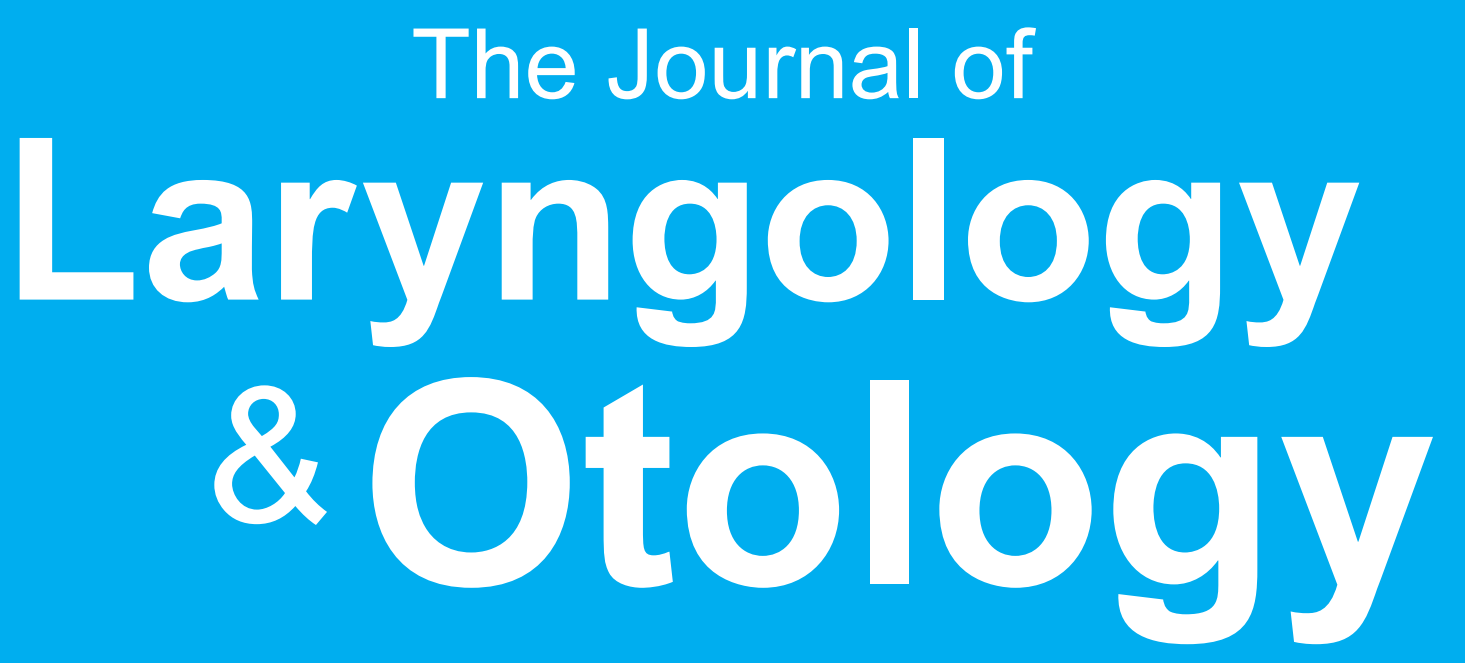

\title{
Australian Supplement
}

DIGITAL ARCHIVE (1887 TO PRESENT) - FREE TO ALL CURRENT SUBSCRIBERS

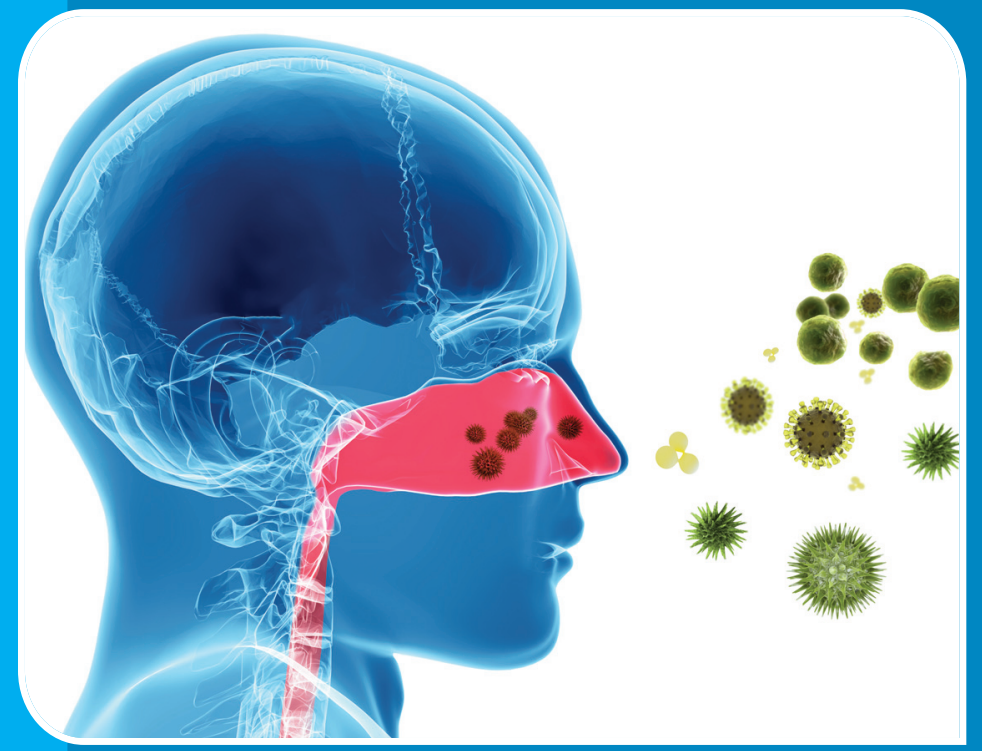

\section{KEY PAPERS}

Computed tomography versus bronchoscopy for foreign bodies Australian training in sleep surgery Coblation therapies for airway stenosis Differential diagnosis in auditory neuropathy

Betadine is ciliotoxic to sinus mucosa
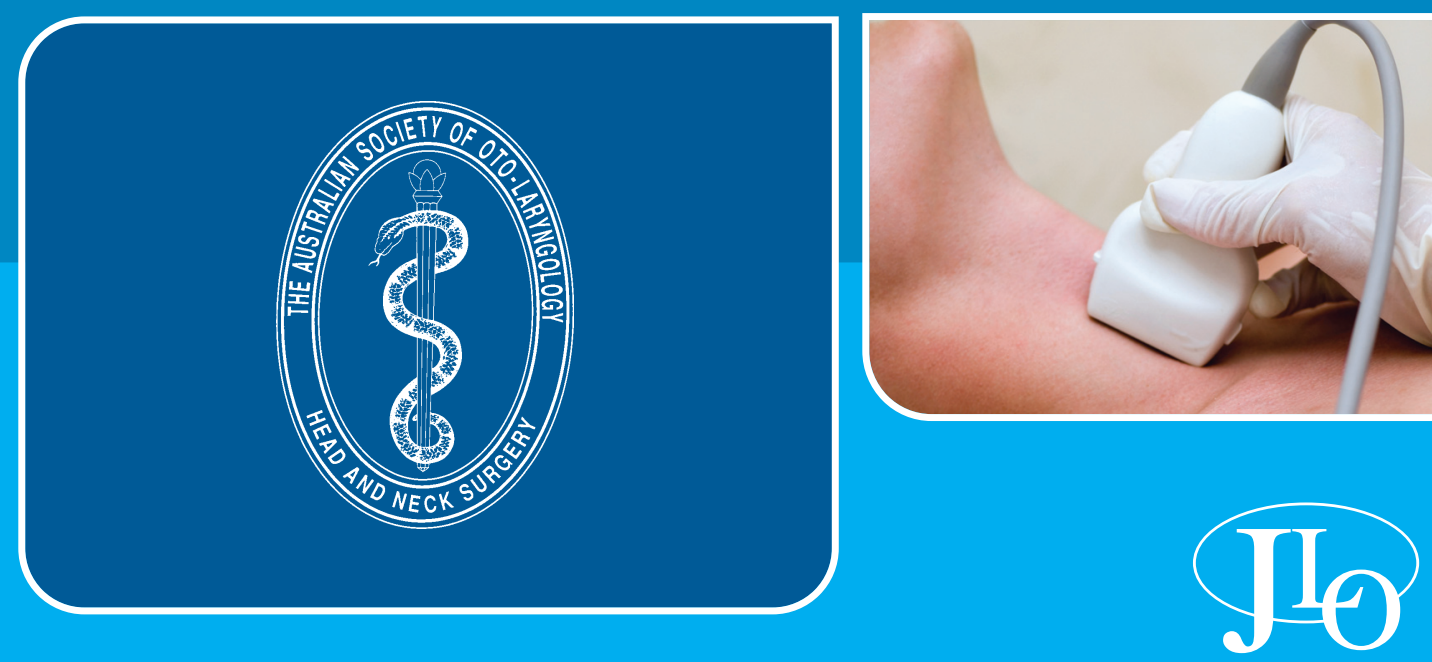

Founded in 1887 


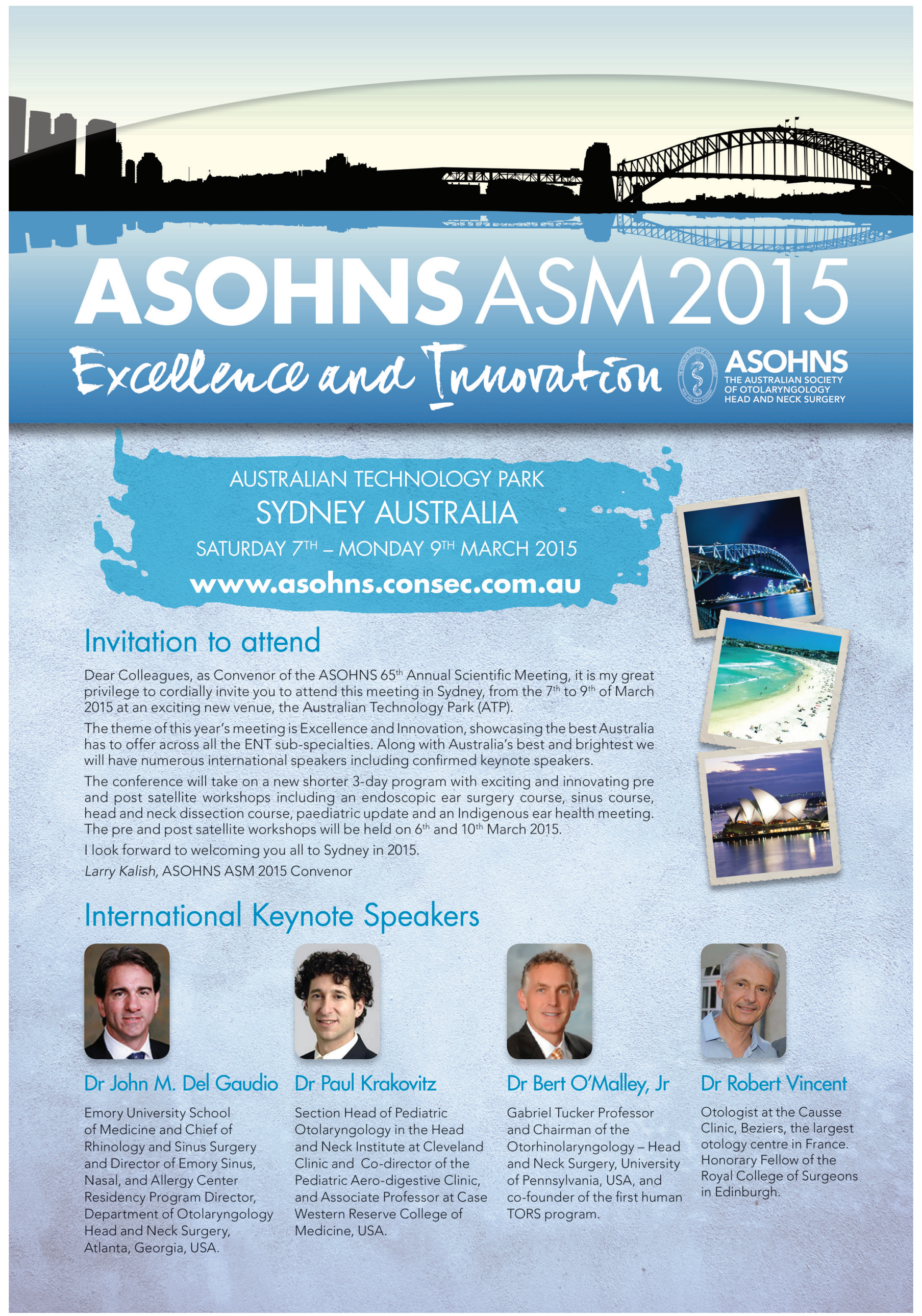




\section{Laryngology \& Otology}

Founded in 1887 by Morell Mackenzie and Norris Wolfenden

\section{Australian Supplement}

\section{Australian Supplement Editor}

Richard Harvey (Sydney)

\author{
Australian Supplement Editorial Board \\ Marcus D. Atlas (Perth) \\ Robert Briggs (Melbourne) \\ Anders Cervin (Brisbane) \\ Richard G. Douglas (Auckland) \\ Peter Friedland (Perth) \\ Richard M. Gallagher (Sydney) \\ Scott M. Graham (Iowa City) \\ Richard Harvey (Sydney) \\ Stephen O'Leary (Melbourne) \\ Benedict J. Panizza (Brisbane) \\ Alkis Psaltis (Adelaide) \\ Raymond Sacks (Sydney) \\ Sanjai Sood (Leeds) \\ Editors \\ Robin Youngs (Gloucester) \\ Edward Fisher (Birmingham) \\ Emeritus Editor \\ Guy Kenyon (London) \\ Senior Assistant Editors \\ Liam Flood (Middlesbrough) \\ Quentin Gardiner (Dundee) \\ Vinidh Paleri (Newcastle)
}

\section{Assistant Editors}

Kim Ah-See (Aberdeen)

Martin Bailey (London)

David Baguley (Cambridge)

Jon Bennett (Plymouth)

Abir Bhattacharyya (London)

Brian Bingham (Glasgow)

Sean Carrie (Newcastle)

Kate Evans (Gloucester)

Jonathan M. Fishman (London)

Charlie Hall (Gloucester)

\author{
Omar Hilmi (Glasgow) \\ Claire Hopkins (London) \\ S S Musheer Hussain (Dundee) \\ Richard Irving (Birmingham) \\ Hisham Khalil (Plymouth) \\ Bhik Kotecha (London) \\ Tristram Lesser (Liverpool) \\ Valerie Lund (London) \\ Ann-Louise McDermott (Birmingham) \\ Robert Mills (Khon-kaen) \\ Desmond Nunez (Bristol) \\ Andrew Prichard (Shrewsbury) \\ Peter Robb (Epsom) \\ Mark Samaha (Quebec) \\ Azhar Shaida (London) \\ Nick Stafford (Hull) \\ lain Swan (Glasgow) \\ John Watkinson (Birmingham) \\ Richard Wight (Middlesbrough) \\ Tim Woolford (Manchester) \\ Matthew Yung (Ipswich)
}

Advisers in Audiology

Doris-Eva Bamiou (London)

Linda Luxon (London)

Advisers in Pathology

Simon Rose (Bath)

Ketan Shah (Oxford)

Adrian Warfield (Birmingham)

Adviser in Radiology

Tim Beale (London)

Adviser in Statistics

Christopher Palmer (Cambridge)

Website Editor

Stephen Jones

Managing Editor

Rosamund Greensted

Unless indicated otherwise, the papers in this Supplement were solicited by the Australian Supplement Editor and approved by the Editors of The Journal of Laryngology \& Otology. They were reviewed by the Australian Supplement Editor, and have undergone the standard journal formal peer review process. All papers may be cited; where content has been previously published in a regular Issue of the Journal, citations should be to the original Volume and Issue of publication (see individual paper details, and Table of Contents), and not to this Supplement.

Cover images: (Upper left) 3d rendering illustration of pollen or virus (C) goa novi /shutterstock; (Lower right) scanning of a thyroid of man (C) Bork /shutterstock

Editorial Office: Maybank, Quickley Rise, Chorleywood, Herts WD3 5PE, UK.

Tel/fax: +44 (0)1923 283561. Email: j.1.o@btconnect.com. Website: http://www.jlo.co.uk

The Journal of Laryngology \& Otology (ISSN 0022-2151) is published monthly in both print and electronic form and distributed on behalf of the proprietors, JLO (1984) Ltd, by Cambridge University Press

Registered Charity No 293063 